Draft Version FEBRUARY 16, 2021

Typeset using IATEX twocolumn style in AASTeX63

\title{
Possible Periodic Dips in the Pulsating Ultraluminous X-ray Source M51 ULX-7
}

\author{
Chin-Ping Hu, ${ }^{1,2, *}$ Yoshiniro Ueda, ${ }^{2}$ And Teruaki Enoto ${ }^{3}$ \\ ${ }^{1}$ Department of Physics, National Changhua University of Education, Changhua, 50007, Taiwan \\ ${ }^{2}$ Department of Astronomy, Kyoto University, Kitashirakawa-Oiwake-cho, Sakyo-ku, Kyoto 606-8502, Japan \\ ${ }^{3}$ Extreme Natural Phenomena RIKEN Hakubi Research Team, Cluster for Pioneering Research, RIKEN, 2-1 Hirosawa, Wako, Saitama \\ 351-0198, Japan
}

(Accepted Dec. 29, 2020)

\begin{abstract}
We report the discovery of possible periodic X-ray dips in a pulsating ultraluminous X-ray source, M51 ULX-7, with the archival Chandra observations. With $\sim 20$ days of monitoring in the superorbital descending state, we discovered three dips with separations of $\sim 2$ and $\sim 8$ days via the Bayesian block technique. A phase-dispersion minimization and a $\chi^{2}$ test suggest that the dip is likely recurrent with a period of $\sim 2$ days, consistent with the orbital period of M51 ULX-7. We interpret the dip as an obscuring of the emission from the pulsar by the vertical structure on the stream-disk interaction region or the atmosphere of the companion star. Both interpretations suggest the viewing angle to be $\sim 60$ degrees. Given that the magnetic field of M51 ULX-7 is moderately high, $B \sim 10^{13} \mathrm{G}$, a low geometric beaming with $b \lesssim 1 / 2$ is sufficient to explain the observed flux and the presence of dips. Obscuration of the stellar wind remains an alternative possible origin and further monitoring of the dips will be required.
\end{abstract}

Keywords: Neutron Stars (1108); X-ray binnary stars (1811); X-ray sources (1822)

\section{INTRODUCTION}

Ultraluminous X-ray sources (ULXs) are extragalactic off-nuclear X-ray point sources with luminosities exceeding the Eddington limit of a stellar-mass black hole (BH) (Feng \& Soria 2011; Kaaret et al. 2017). They are the most extreme populations of compact objects that break several theoretical limits. The most remarkable one is the Eddington limit, which can be calculated as $L_{\mathrm{Edd}}=4 \pi G M m_{\mathrm{p}} / \sigma_{\mathrm{T}} \approx 1.3 \times 10^{38}\left(M / M_{\odot}\right) \mathrm{erg} \mathrm{s}^{-1}$, where $\sigma_{\mathrm{T}}$ is the Thomson scattering cross-section, $m_{\mathrm{p}}$ is the mass of a proton, and $M$ is the mass of the accretor. For spherical accretion, this is the upper limit of the luminosity because the strong radiation pressure prevents mass accretion. Intermediate-mass $\mathrm{BHs}$ with masses of $10^{2}-10^{4} M_{\odot}$ could explain the observed high luminosity but only a few ULXs are candidates for intermediate-mass BHs (Godet et al. 2012; Straub et al. 2014). Alternatively, most ULXs are likely stellar-mass compact objects with super-Eddington accretion rates and mild beaming through population studies (Kaaret et al. 2017).

Corresponding author: C.-P. Hu

cphu0821@cc.ncue.edu.tw

* JSPS International Research Fellow
The discovery of pulsating ULXs (PULXs) is a milestone that challenges current understanding of both the ULXs and the magnetospheric accretion of neutron stars (NSs). Until early 2020, eight PULXs have been discovered (Trudolyubov et al. 2007; Trudolyubov 2008; Bachetti et al. 2014; Fürst et al. 2016; Israel et al. 2017; Carpano et al. 2018; Sathyaprakash et al. 2019; Rodríguez Castillo et al. 2020). The Eddington luminosity of an NS is one order of magnitude lower than that of a $10 M_{\odot}$ BH. PULXs are believed to be powered by NSs with extremely high mass accretion rates and/or extremely high magnetic fields. It was proposed that an NS with an extremely high magnetic field could explain the observed luminosity because the cross-section can be reduced and the emission can radiate along the direction perpendicular to the accreting flow (Basko \& Sunyaev 1975; Mushtukov et al. 2015). Alternatively, a strong magnetic field may not be necessary if their emission could be more strongly collimated compared to ULXs that harbor BHs (King \& Lasota 2019). Most of the currently known PULXs can be well interpreted with the super-critical accretion model in which the emission is highly beamed toward the Earth (King \& Lasota 2019). For example, the half-opening angle of the funnel and the inclination angle of NGC $5907 \mathrm{X}-1$ are estimated as $\lesssim 10^{\circ}$ (Dauser et al. 2017). 
M51 is an interacting galaxy pair consisting of an active face-on spiral galaxy, NGC 5194, and a dwarf galaxy, NGC 5195. This system contains hundreds of X-ray sources including at least nine ULXs with $L_{X}>$ $10^{39} \mathrm{erg} \mathrm{s}^{-1}$ and 13 point X-ray sources with $10^{38} \mathrm{erg}$ $\mathrm{s}^{-1}<L_{X}<10^{39} \mathrm{erg} \mathrm{s}^{-1}$ (Terashima \& Wilson 2004). M51 ULX-7 (also known as CXOU J133001.0+471344 and RX J133001+47137, hereafter ULX-7) is a highly variable ULX located at a spiral arm toward the northwest from the center of NGC 5194. ULX-7 was recently found to be a PULX with a spin period of 2.8 $\mathrm{s}$ and an orbital period of $\sim 2$ days. The inclination angle is unconstrained due to the lack of X-ray orbital modulations in XMM-Newton data (Rodríguez Castillo et al. 2020). A superorbital modulation with a period of $\approx 38$ days can be observed with the monitoring data taken by the Neil Gehrels Swift observatory although the mechanism remains unknown (Brightman et al. 2020; Vasilopoulos et al. 2020). The measured spin-up rate $\left(\dot{\nu}=3.1(8) \times 10^{-11} \mathrm{~Hz} \mathrm{~s}^{-1}\right)$ suggests that the NS in M51 ULX-7 has a moderately strong magnetic field with $B=10^{12}-10^{13} \mathrm{G}$ assuming that the superorbital modulation is caused by changes in the mass accretion rate (Rodríguez Castillo et al. 2020), or $B=(3-$ 4) $\times 10^{13} \mathrm{G}$ if the superorbital modulation is caused by the free precession of the NS (Vasilopoulos et al. 2020).

\section{OBSERVATIONS AND DATA REDUCTION}

\subsection{Chandra}

M51 was observed with the Advanced CCD Imaging Spectrometer (ACIS) of Chandra 16 times between 2000 and 2018. Except for ObsID 19522 which was observed with ACIS-I chips, they were observed with ACIS-S chips. Seven of them (ObsIDs: C1: 13813, C2: 13812, C3: 15496, C4:13814, C5: 13815, C6: 13816, and C7: 15553) were intensively carried out between 2012 September and 2012 October (see Table 1). These data sets allow us to investigate variability with time scales from a few hours to a few days. We reprocess all of the data sets using the pipeline chandra_repro in the Chandra Interactive Analysis of Observations (CIAO) version 4.9 with the calibration database (CALDB) version 4.7.3 (Fruscione et al. 2006).

We extract source events from a circular aperture of $3^{\prime \prime}$ radius in which $95 \%$ of the source photons are encircled. We use dmextract to extract the light curve of ULX-7 in $0.5-7 \mathrm{keV}$ with $7200 \mathrm{~s}$ time resolution. The background is extracted from a nearby source-free region and subtracted from the source light curve. The time intervals with background flares are removed by using the 1c_clean command. We find that the count rate sometimes falls to zero or rises to extremely large values at the beginning or end of an observation. Therefore, we remove the boundary points of each observation.

\subsection{XMM-Newton}

XMM-Newton has carried out 10 observations targeting M51 before 2019, while four of them (ObsIDs X1: 0824450901, X2: 0830191401, X3: 0830191501, and X4: 0830191601) with long exposure times were made in 2018 May and June. All the EPIC cameras were operated in the full-frame mode in $\mathrm{X} 1$, while the MOS camera switched to small window mode in the remaining three observations to resolve the pulsation signal of ULX-7. We downloaded the data from the $X M M-N e w t o n$ Science Archive. The pipeline procedures epproc and emproc tasks in the XMM-Newton Science Analysis Software (SAS version 18.0.0) are used to reprocess the PN and MOS data with the latest calibration files, respectively. We do not further filter out any time intervals since no significant background flaring was found in these observations.

We merge the events from all EPIC cameras to increase the signal-to-noise ratio and extract $X M M$ Newton light curves of ULX-7 using the epiclccorr command. The source events are selected from a $15^{\prime \prime}$ circle centered on ULX-7. This criterion only encircles $\lesssim 70 \%$ of source energy, but can help to avoid contamination from a nearby source, CXOU J133004.3+471321, which is only $40^{\prime \prime}$ away from ULX-7. The background is extracted from a source-free region although contamination from diffuse emission from the spiral arm of M51a remains possible. The time bin size of the light curve is set to $3600 \mathrm{~s}$.

\section{ANALYSIS AND RESULTS}

\subsection{Chandra Light Curves}

Following the standard analysis procedure described in Section 2, we create background-subtracted light curves with a bin size of two hours in the $0.5-7 \mathrm{keV}$ band (see Figure $1(\mathrm{a})$ ). The count rate decreases from 0.06 counts $\mathrm{s}^{-1}$ to almost zero. This is likely the transition from high to low states in a superorbital cycle. Three dips are seen in observations C1 (dip1), C2 (dip2), and C4 (dip3). Dip 1 and dip 3 are fully observed, while dip 2 is partially seen.

To test whether the dips are statistically significant, we utilize the Bayesian block ${ }^{1}$ technique to search for significant flux variability (Scargle et al. 2013). Data sets C5 and C6 are not included in this analysis because they are likely observed in the superorbital low low sate. The flux variability in other data sets is heavily dominated by the flux decrease in the superorbital cycle. We normalize the count rate of each observation with respect to the superorbital decreasing trend, which is obtained by smoothing the light curve in Figure 1(a), to make the average value to be unity. We also tried to obtain the trend individually by fitting each data set with a linear function and obtaining a consistent result.

\footnotetext{
${ }^{1}$ https://docs.astropy.org/en/stable/api/astropy.stats.bayesian_ blocks.html
} 
Table 1. Data sets used in this work.

\begin{tabular}{|c|c|c|c|c|c|c|}
\hline Epoch & Observatory & ObsID & Instrument & Mode & Start Date & Exposure (ks) \\
\hline $\mathrm{C} 1$ & Chandra & 13813 & ACIS-S & $\mathrm{TE}$ & 2012-09-09 17:47:30 & 179.2 \\
\hline $\mathrm{C} 2$ & Chandra & 13812 & ACIS-S & $\mathrm{TE}$ & 2012-09-12 18:23:50 & 157.5 \\
\hline C3 & Chandra & 15496 & ACIS-S & $\mathrm{TE}$ & 2012-09-19 09:20:34 & 41.0 \\
\hline $\mathrm{C} 4$ & Chandra & 13814 & ACIS-S & $\mathrm{TE}$ & 2012-09-20 07:21:42 & 189.9 \\
\hline C5 & Chandra & 13815 & ACIS-S & $\mathrm{TE}$ & 2012-09-23 08:12:08 & 67.2 \\
\hline C6 & Chandra & 13816 & ACIS-S & $\mathrm{TE}$ & 2012-09-26 05:11:40 & 73.1 \\
\hline $\mathrm{C} 7$ & Chandra & 15553 & ACIS-S & $\mathrm{TE}$ & 2012-10-10 00:43:36 & 37.6 \\
\hline \multirow[t]{3}{*}{$\mathrm{X} 1$} & XMM-Newton & 0824450901 & $\mathrm{PN}$ & $\mathrm{FF}$ & 2018-05-13 22:02:37 & 74.8 \\
\hline & & & MOS1 & $\mathrm{FF}$ & 2018-05-13 21:36:55 & 76.6 \\
\hline & & & MOS2 & $\mathrm{FF}$ & 2018-05-13 21:37:15 & 76.6 \\
\hline \multirow[t]{3}{*}{$\mathrm{X} 2$} & XMM-Newton & 0830191401 & $\mathrm{PN}$ & $\mathrm{FF}$ & 2018-05-25 21:10:48 & 94.8 \\
\hline & & & MOS1 & SW & 2018-05-25 20:45:06 & 96.6 \\
\hline & & & MOS2 & SW & 2018-05-25 20:45:28 & 96.6 \\
\hline \multirow[t]{3}{*}{ X3 } & XMM-Newton & 0830191501 & PN & FF & 2018-06-13 02:22:52 & 59.8 \\
\hline & & & MOS1 & SW & 2018-06-13 01:57:11 & 61.6 \\
\hline & & & MOS2 & SW & 2018-06-13 01:57:33 & 61.6 \\
\hline \multirow[t]{3}{*}{$\mathrm{X} 4$} & $X M M-N e w t o n$ & 0830191601 & $\mathrm{PN}$ & $\mathrm{FF}$ & 2018-06-15 02:08:11 & 59.8 \\
\hline & & & MOS1 & SW & 2018-06-15 01:42:29 & 61.6 \\
\hline & & & MOS2 & SW & 2018-06-15 01:42:51 & 61.6 \\
\hline
\end{tabular}

The abbreviations and time resolution of the observation mode are described as follows. TE: timed exposure mode of full-frame ACIS-S chip has a time resolution of $3.2 \mathrm{~s}$. FF: full-frame mode with time resolution of $73.4 \mathrm{~ms}$ for PN and $2.6 \mathrm{~s}$ for MOS. SW: small window mode of the MOS camera with time resolution of $0.3 \mathrm{~s}$.

With a false alarm probability of $p_{0}=0.003$, we identified four significant flux variations. Three of them (i, ii, and iii in Figure 1) correspond to the visible dips, implying that the flux of these dips significantly deviates from the long-term flux trend. The center time of dip 1 and dip 3 are simply calculated from the center of the blocks as MJD 56180.99 and 56191.06, respectively. The fourth (iv) flux variation corresponds to a sudden flux increment in the last time bin of the $\mathrm{C} 4$ observation. This could be caused by flares of ULX-7 or unknown instrumental effects, which is beyond the scope of this paper.

As an alternative method to estimate dip parameters, we fit the count rate during the non-dip state with a straight line and estimate the dip center time $\left(t_{\mathrm{c}}\right)$ as the summation of the time of all the bins in the dip with a weighting factor defined as the difference between measured count rate and estimated non-dip count rate $(\mathrm{Hu}$ et al. 2008). We find that $t_{(\mathrm{c}, \text { dip } 1)}=$ MJD $56180.99 \pm 0.03$ and $t_{(\mathrm{c}, \mathrm{dip} 3)}=$ MJD $56191.06 \pm 0.03$, consistent with those directly obtained from the blocks. Dip 2 is not fully observed but the center is likely located around MJD 56183. The time separation between dip 1 and 2 is two days and that between dip 2 and dip 3 is eight days. Given that the orbital period of M51 ULX-7 is two days, we suggest that this dip feature appeared periodically per binary orbit. The widths of the dips are calculated as the dispersion, i.e., the second moment of the dip state, as $W_{\text {dip } 1}=0.07 \pm 0.03$ days and $W_{\text {dip } 3}=0.16 \pm 0.02$ days. This hints that the width could be superorbital phase-dependent.

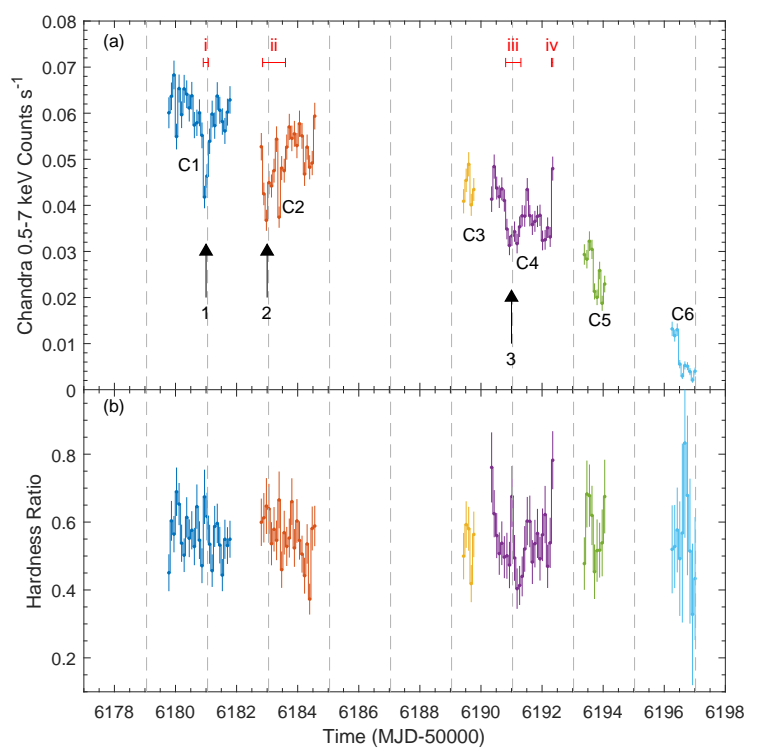

Figure 1. (a) The Chandra $0.5-7 \mathrm{keV}$ light curve of ULX-7 observed in 2012. Six data sets (C1-C6) are plotted. This 20 day light curve was carried out during the descent of the superorbital modulation. Three dips occurred at MJD 56181, 56183, and 56191, indicated by arrows. The gray dashed line denotes the expected dip arrival times by assuming that the dip arrives periodically with a period equal to the orbital period of 1.9969 days. The intervals i-iv indicate the time intervals during which the Bayesian block analysis identifies significant flux deviation from the long-term trend. (b) Corresponding hardness ratio curve of ULX-7. 

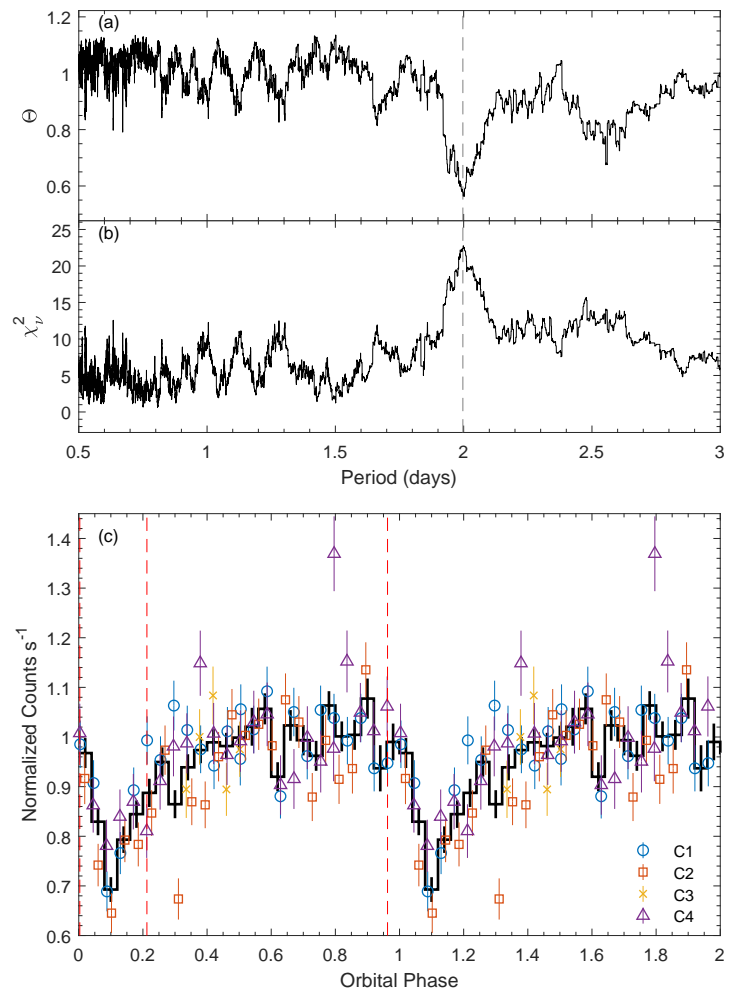

Figure 2. (a) Phase dispersion minimization and (b) the epoch folding period search results of the normalized Chandra light curve of ULX-7. The vertical dashed line is the orbital period derived from the orbital Doppler effect of the pulsar (Rodríguez Castillo et al. 2020). (c) Normalized Chandra light curve folded with the orbital period of ULX-7. The scattered data points represent data points from data sets $\mathrm{C} 1-\mathrm{C} 4$, while the black histogram denotes the mean orbital profile by dividing an orbital cycle into 24 bins. A dip can be seen at orbital phase $\phi_{\text {orb }} \approx 0.1$. The red dashed lines are edges where the Bayesian block technique identifies a significant change in the count rate.

To test whether the dip originates from the orbital motion, we searched for periodicity with two non-Fourierbased algorithms, the phase dispersion minimization (Stellingwerf 1978) and the epoch folding period search (Leahy et al. 1983) applied to the detrended Chandra light curve of ULX-7. The result is shown in Figure 2. Both algorithms show a signal around the known orbital period of $\sim 2$ days (Rodríguez Castillo et al. 2020).

To test whether the spectral behavior of ULX-7 changes during the dip, we calculate the hardness ratio $(H R)$ defined as $H R=\left(\mathrm{r}_{\mathrm{h}}-\mathrm{r}_{\mathrm{s}}\right) /\left(\mathrm{r}_{\mathrm{h}}+\mathrm{r}_{\mathrm{s}}\right)$, where $\mathrm{r}_{h}$ is the count rate in the $2-7 \mathrm{keV}$ band and $\mathrm{r}_{s}$ is that in the count rate at $0.5-2 \mathrm{keV}$ (see Figure 1 (b)). We find no clear hardening during the dip, similar to a few other dipping systems in the same galaxy (Urquhart \& Soria 2016; Wang et al. 2018).
We further folded the light curve with the binary orbital period to obtain the orbital profile. The phase zero epoch is set to MJD 58285.0084 (2018 June 16), which is the superior conjunction when the pulsar is behind the companion star. The unbinned light curve and binned orbital profile are plotted in Figure 2 (c). A clear orbital dip can be seen with a dip center phase at 0.1 . To test whether the dip is significantly determined, we again used the Bayseian block technique to search for significant variability in the unbinned folded light curve (see the colored data points in Figure 2). We searched for optimized blocks by setting the false alarm probability $p_{0}$ to be 0.003 . The result shows that an entire orbital cycle can be divided into two blocks, including a dip at $\phi_{\text {orb }}<0.21$ and a persistent phase at $\phi_{\text {orb }}>0.21$

\subsection{Chandra Spectral Analysis}

We then investigate X-ray spectral behaviors during the dip states and the non-dip states. We use dmgti to split the event files into the dip and non-dip states, and use specextract to extract the X-ray source and background spectra and corresponding response files and ancillary files. The spectral fitting is achieved using CIAO's modeling package SHERPA. We fit the $0.3-$ $10 \mathrm{keV}$ spectra using the Cash statistics (Cash 1979). We choose the absorption model tbabs and the corresponding solar abundance (Wilms et al. 2000). For the C6 observation, we extract the spectrum before the flux drops to almost zero. We fit the spectra with an absorbed power law (Earnshaw et al. 2016). The hydrogen column density $N_{\mathrm{H}}$ is consistent with $\approx 10^{21} \mathrm{~cm}^{-2}$ except for that in the $\mathrm{C} 6$ observation. The photon indices are consistent with $\Gamma \approx 1.5$ in all the data sets except for the dip phase in the $\mathrm{C} 1$ observation. The photon indices obtained in the non-dip states of all observations are fully consistent with those presented in Earnshaw et al. (2016). The best-fit parameters are presented in the Table 2 .

We calculate the absorbed model flux in the soft band $(0.3-2 \mathrm{keV})$ and hard band $(2-10 \mathrm{keV})$ using the sample_flux command. Then we calculate the ratio between the flux at the hard band and the flux at the soft band. The ratio of the non-dip state is stable at $\approx 2.8$ and likely X-ray flux independent. All the ratios determined in the dip states remain consistent with those in the non-dip states although the uncertainties are clearly large.

In the $\mathrm{C} 7$ observation, an excess of $\mathrm{X}$-ray photons can still be seen in the position of ULX-7. The spectral parameters cannot be constrained if we set all parameters to be free. After freezing $N_{\mathrm{H}}$ to be $1.1 \times 10^{21} \mathrm{~cm}^{-2}$, the photon index is constrained as $\Gamma=3 \pm 1$ with a statistic of $C^{2} / d o f=13.8 / 20$. The X-ray luminosity can be estimated as $5_{-2}^{+4} \times 10^{37}$ erg s$^{-1}$, which can be considered as the upper limit of the flux in the superorbital low state.

We noticed that spectra of PULXs can usually be fit with complex models containing more than one compo- 

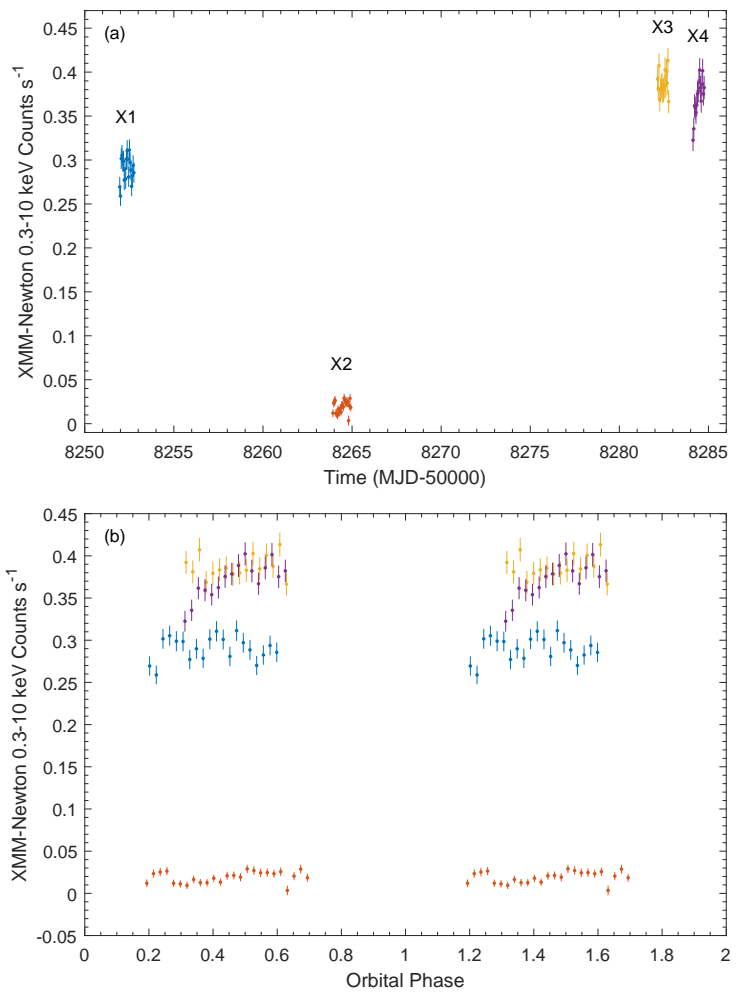

Figure 3. (a) XMM-Newton $0.3-10 \mathrm{keV}$ light curve of M51 ULX-7. The epochs of individual data sets are labeled. (b) Light curve folded with the orbital ephemeris (Rodríguez Castillo et al. 2020). The orbital phases $0.7-1.2$ are not covered by XMM-Newton observations.

nent (see, e.g., Koliopanos et al. 2017). Detailed spectral analysis with the XMM-Newton data sets of X1 - X4 suggests that the soft X-ray emission of ULX-7 can be empirically described by a low-temperature disk blockbody plus a high-temperature blackbody (Rodríguez Castillo et al. 2020). We use this two-component model to fit the Chandra spectra of ULX-7 during non-dip states by linking $N_{\mathrm{H}}$ across all observations. The spectra can be fit with an inner disk temperature of $k T_{\text {in }} \approx 0.36$ $\mathrm{keV}$ and a hot blackbody with a temperature $k T_{\mathrm{BB}} \approx$ $1.1 \mathrm{keV}$ across all the observations except for C6 which contains large uncertainties. Alternatively, the spectra can also be equally well described with other models, e.g., a cool blackbody plus a hot disk blackbody, or a cool disk blackbody plus a Comptonized corona. The implications of detailed spectroscopy, which is beyond the scope of this paper, have been discussed in Rodríguez Castillo et al. (2020). We attempted to fit the dip spectra with these two-component models, but did not observe significant differences in spectral parameters compared to non-dip spectra.

\subsection{XMM-Newton Light Curves}

Figure 3 (a) shows the XMM-Newton light curves using observations described in Table 1 of ULX-7 in 0.3
$-10 \mathrm{keV}$. X2 is likely observed in the superorbital low state, while the other three are taken in high or transition states. We observe no clear dip in the light curve. We then fold the light curve according to the orbital ephemeris and find that these four observations only cover orbital phases $0.2-0.7$ (see Figure 3 (b)). This is expected because the orbital period of XMM-Newton in 2018 is $47.86 \mathrm{hr}$ (1.9942 days), which is very close to the orbital period of ULX-7 (1.9969 days). Given that only $\sim 40 \mathrm{hr}$ are available for observation in each XMMNewton orbit, it is impossible for the orbital phase of ULX-7 to be fully covered in a $\sim 40$-day time span. Nevertheless, the XMM-Newton observation suggests that the dips are unlikely to occur at orbital phases $0.2-0.7$, implying that the dips may occur near the phase of the superior conjunction of the pulsar.

\section{DISCUSSION}

The dips in Roche-lobe-filled X-ray binary systems are believed to be caused by an obscuring effect. The $\mathrm{X}$-ray emission from the compact object and the inner accretion disk is obscured by the bulge on the rim of the accretion disk or the ring on the circularization radius (Naik et al. 2011). The absorption is strong in the soft X-ray band and a spectral hardening is expected. Currently known PULXs with short orbital and superorbital periods are likely driven by the Rochelobe overflow although some wind-driven models are not entirely excluded (Rodríguez Castillo et al. 2020; Townsend \& Charles 2020). These Roche-lobe overflow high-mass X-ray binaries (HMXBs) could show orbitalphase-dependent dips that can be interpreted as the obscuring effect by the bulge similar to low-mass X-ray binaries (Naik et al. 2011; Hu et al. 2013). In addition, the large size and the extended atmosphere of the companion star in a wind-fed HMXB make it possible to have an atmospheric eclipse that behaves like a dip and the X-rays from the NS are not fully obscured (Grundstrom et al. 2007; Hu et al. 2017). A high inclination angle is needed for both scenarios. However, the lack of a hardness change in ULX-7 during the dips is puzzling. This could be interpreted as a result of insufficient photon statistics, partial covering of the extended soft X-ray emission, or an extremely high optical depth of the absorber in the Chandra energy band. Given that the disk and the disk wind could contribute supersoft emission from a more extended region compared to the pulsed emission, a softening during the dip is possible if the supersoft emission is less obscured than the hard emission coming from the NS (Zhou et al. 2019). This partial covering of an extended soft emitter is also used to interpret the lack of hardness ratio change in NGC 55 X-1 (Stobbart et al. 2004), although the emission mechanism for the soft emission in ULX-7 is quite different (Stobbart et al. 2004). Another possible scenario for the dip is the intrinsic variability of the mass accretion rate although this is not likely orbital dependent. 
Table 2. Best-fit parameters of Chandra spectra of M51 ULX-7 with a single power law.

\begin{tabular}{cccccccccc}
\hline \hline Epoch & State & Counts & $N_{\mathrm{H}}$ & $\Gamma$ & $L_{0.3-10 \mathrm{keV}}$ & $F_{0.3-2 \mathrm{keV}}$ & $L_{2-10 \mathrm{keV}}$ & Ratio & $C^{2} /$ dof \\
\hline C1 & Non-dip & 9047 & $1.3 \pm 0.2$ & $1.50 \pm 0.03$ & $76 \pm 3$ & $17.4 \pm 0.7$ & $50 \pm 3$ & $2.8 \pm 0.2$ & $508.2 / 498$ \\
C1 & Dip & 1021 & $0.6_{-0.3}^{+0.5}$ & $1.32 \pm 0.07$ & $60_{-7}^{+8}$ & $13 \pm 2$ & $45 \pm 7$ & $3.3 \pm 0.5$ & $284.7 / 320$ \\
C2 & Non-dip & 4208 & $1.1 \pm 0.2$ & $1.54 \pm 0.05$ & $63 \pm 3$ & $16 \pm 1$ & $40 \pm 3$ & $2.6 \pm 0.3$ & $402.2 / 442$ \\
C2 & Dip & 3340 & $1.2 \pm 0.2$ & $1.48 \pm 0.05$ & $56 \pm 4$ & $13 \pm 1$ & $37 \pm 3$ & $2.9 \pm 0.3$ & $428.6 / 425$ \\
C3 & Non-dip & 1827 & $0.8 \pm 0.3$ & $1.47 \pm 0.06$ & $53 \pm 5$ & $13 \pm 1$ & $36 \pm 4$ & $2.8 \pm 0.4$ & $375.3 / 377$ \\
C4 & Non-dip & 4629 & $1.6 \pm 0.3$ & $1.53 \pm 0.04$ & $50 \pm 3$ & $11 \pm 1$ & $33 \pm 3$ & $3.0 \pm 0.3$ & $457.4 / 449$ \\
C4 & Dip & 1986 & $1.4 \pm 0.3$ & $1.68 \pm 0.06$ & $40 \pm 4$ & $10 \pm 1$ & $24 \pm 3$ & $2.4 \pm 0.4$ & $364.6 / 377$ \\
C5 & Non-dip & 1745 & $1.1 \pm 0.3$ & $1.51 \pm 0.07$ & $31 \pm 3$ & $7.5 \pm 0.8$ & $20 \pm 3$ & $2.8 \pm 0.4$ & $323.7 / 371$ \\
C6 & Non-dip & 479 & $<0.3$ & $1.4 \pm 0.1$ & $7.4_{-0.8}^{+0.9}$ & $2.0 \pm 0.2$ & $5.1 \pm 0.9$ & $2.5 \pm 0.5$ & $239.8 / 224$ \\
\hline
\end{tabular}

The unit of $N_{\mathrm{H}}$ is $10^{21} \mathrm{~cm}^{-2}$; the unit of the unabsorbed luminosity $L_{0.3-10 \mathrm{keV}}$ is $10^{38} \mathrm{erg} \mathrm{s}^{-1}$; the unit of the absorbed flux is $10^{-13}$ erg $\mathrm{s}^{-1} \mathrm{~cm}^{-2}$. The column counts is the number of source counts, where the estimated background counts are subtracted.

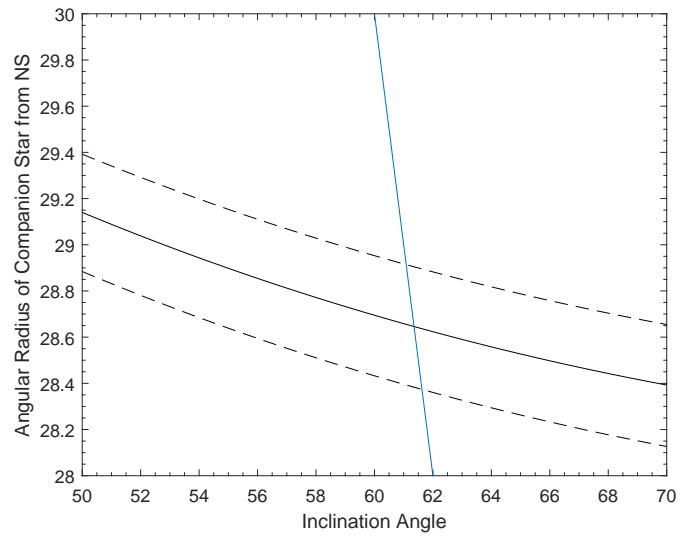

Figure 4. Black line: angular size $\phi$ of the companion star as a function of the inclination angle $i$ under the assumption that the companion star fills its Roche lobe. Dashed lines: 1- $\sigma$ intervals. Blue line: critical value for the Companion star to pass through the line of sight.

If we further assume that the complement of the inclination angle $(i)$ is not very far from the angular size of the companion star, the inclination angle could be constrained. The effective Roche-lobe size $\left(r_{\mathrm{L}}\right)$ can be estimated as

$$
\frac{r_{\mathrm{L}}}{a}=\frac{0.49 q^{2 / 3}}{0.6 q^{2 / 3}+\ln \left(1+q^{1 / 3}\right)}
$$

where $q$ is the mass ratio and $a$ is the semi-major axis of the orbit of the compact object (Eggleton 1983). Since the mass function and the semi-major axis of ULX-7 have been derived with pulsar timing as a function of $i$ (see Rodríguez Castillo et al. 2020), $r_{\mathrm{L}}$ as well as the angular size of the companion star $(\phi)$ are functions of $i$ (see Figure 4). The blue line in Figure 4 denotes $i=$ $90^{\circ}-\phi$, which is the critical value of the occurrence of the eclipse. The intersection of $\phi(i)$ and this blue line suggest that the critical value of the inclination angle is $\sim 62^{\circ}$, which is the upper limit of the inclination angle of ULX-7. Since we did not observe total eclipse, the inclination angle is likely slightly lower than this critical value (Figure 4).

A moderate geometric beamed funnel with a halfopening angle of $\sim 60^{\circ}$ could explain the observed luminosity. This could also explain the presence of dips and the precession of the funnel could explain the superorbital modulation (Rodríguez Castillo et al. 2020). Assuming that the beamed emission is symmetric to the disk plane, the $60^{\circ}$ half-opening angle of each side corresponds to a total solid angle of $2 \pi$, which is half of the entire sphere. This enhances the observed flux by a factor of two compared to an isotropic emitter with the same accretion luminosity. The beaming factor can be derived as $b \equiv L_{\text {acc }} / L_{\text {iso }} \gtrsim 1 / 2$, where $L_{\text {acc }}$ is the total accretion luminosity and $L_{\text {iso }}$ is the observed luminosity by assuming isotropic emission (King 2008). This implies a peak column accretion luminosity of $L_{\mathrm{acc}, \max } \approx 4 \times 10^{39}$ $\operatorname{erg~s}^{-1}$. The maximum accretion luminosity can be approximated as

$$
L_{\text {acc }, \max } \approx 0.35 \times\left(\frac{B}{10^{12} \mathrm{G}}\right)^{3 / 4} \times 10^{39} \mathrm{erg} \mathrm{s}^{-1},
$$

where $10^{13} \mathrm{G}<B<10^{15} \mathrm{G}$ if the high luminosity is powered by a strong magnetic field (Mushtukov et al. $2015)$. Therefore, a magnetic field of $B \gtrsim 3 \times 10^{13} \mathrm{G}$ is needed for ULX-7. We note that this equation is only an approximation and is based on a few assumptions, e.g., the accretion column height cannot be larger than the NS radius. Thus, equation 2 can only be used as an order of magnitude estimation for the maximum magnetic field. Finally, the spectrum of ULX-7 can be described by two components (Rodríguez Castillo et al. 2020), while the hard one likely represents the pulsed emission. Given that the pulsed emission only contributes $\sim 60 \%$ of the total luminosity, the lower limit of the magnetic field could be as low as $1.5 \times 10^{13} \mathrm{G}$. This remains strong enough to exclude a boost from a pure supercritical accretion model without a strong $\left(>10^{13} \mathrm{G}\right)$ magnetic field. However, the funnel-shaped wind could be misaligned with respect to the normal factor of the disk plane. This makes if difficult to find a one-to-one mapping between the inclination angle and the beaming 
factor in PULXs. Further simulation and observational properties will be needed to constrain their relationship.

It remains possible that the stellar wind of the companion star of ULX-7 plays an important role, and the dip could be an absorption effect of wind clumps similar to those of Cyg X-1 and SMC X-2 (Feng \& Cui 2002; $\mathrm{Li}$ et al. 2016). This type of dip can happen at any orbital phase. If this is the case for ULX-7, the inclination angle cannot be well constrained. The orbital phase distribution of this kind of dip is usually less stable than those originating from the disk rim. Therefore, more observations and statistical analysis of dips in ULX-7 are needed to test their origin and constrain the viewing geometry of ULX-7.

\section{SUMMARY}

Utilizing the archival Chandra data taken in 2012, we find that ULX-7 shows periodic dips in its decreasing trend of a superorbital cycle. Through epoch folding analysis and phase dispersion minimization, we find that the period of the dip is consistent with the orbital period. If the dip is caused by the absorption of the Xray emission by the vertical structure on the streamdisk interaction region or the atmosphere of the companion star, the inclination angle of this system could be constrained as not very far from $\sim 60^{\circ}$. This can be achieved if the magnetic field of the NSb is as high as $10^{13} \mathrm{G}$, which has been inferred from previous studies (Vasilopoulos et al. 2020). An absorption from the clumpy wind may also be responsible for the origin of the dips, but they are expected to be less stable compared to those dips caused by the bulge on the disk. Moreover, the lack of hardening during the dip is puzzling with regard to their origin. More observations are needed to investigate the stability, orbital phase dependence, and the spectral behaviors of the dips.

\section{ACKNOWLEDGEMENTS}

We thank the anonymous reviewer for valuable comments that improved this paper. This research is in part based on the data obtained from the Chandra Data Archive and has made use of the software provided by the Chandra X-ray Center (CXC) in the application packages CIAO, ChIPS, and Sherpa. This research has used the observations obtained with XMM-Newton and the ESA science mission with instruments and contributions directly funded by the ESA member states and NASA. This research made use of Astropy, a community effort to develop a common core package for astronomy in Python. C.-P.H. acknowledges support from the the Ministry of Science and Technology in Taiwan through grant MOST 109-2112-M-018-009-MY3 and the support from Japan Society for the Promotion of Science (JSPS; ID: P18318).

Facilities: Chandra (ACIS), XMM (MOS, PN)

Software: CIAO (Fruscione et al. 2006), XMMNewton SAS (Gabriel et al. 2004), Astropy(Astropy Collaboration et al. 2013; The Astropy Collaboration 2018a,b)

\section{REFERENCES}

Astropy Collaboration, Robitaille, T. P., Tollerud, E. J., et al. 2013, A\&A, 558, A33, doi: 10.1051/0004-6361/201322068

Bachetti, M., Harrison, F. A., Walton, D. J., et al. 2014, Nature, 514, 202, doi: 10.1038/nature13791

Basko, M. M., \& Sunyaev, R. A. 1975, A\&A, 42, 311

Brightman, M., Earnshaw, H., Fürst, F., et al. 2020, ApJ, 895, 127, doi: 10.3847/1538-4357/ab7e2a

Carpano, S., Haberl, F., Maitra, C., \& Vasilopoulos, G. 2018, MNRAS, 476, L45, doi: 10.1093/mnrasl/sly030

Cash, W. 1979, ApJ, 228, 939, doi: 10.1086/156922

Dauser, T., Middleton, M., \& Wilms, J. 2017, MNRAS, 466, 2236, doi: 10.1093/mnras/stw3304

Earnshaw, H. M., Roberts, T. P., Heil, L. M., et al. 2016, MNRAS, 456, 3840, doi: 10.1093/mnras/stv2945

Eggleton, P. P. 1983, ApJ, 268, 368, doi: 10.1086/160960

Feng, H., \& Soria, R. 2011, NewAR, 55, 166, doi: 10.1016/j.newar.2011.08.002

Feng, Y. X., \& Cui, W. 2002, ApJ, 564, 953, doi: $10.1086 / 324284$
Fruscione, A., McDowell, J. C., Allen, G. E., et al. 2006, Proc. SPIE, 6270, 62701V, doi: 10.1117/12.671760

Fürst, F., Walton, D. J., Harrison, F. A., et al. 2016, ApJL, 831, L14, doi: 10.3847/2041-8205/831/2/L14

Gabriel, C., Denby, M., Fyfe, D. J., et al. 2004, in Astronomical Society of the Pacific Conference Series, Vol. 314, Astronomical Data Analysis Software and Systems (ADASS) XIII, ed. F. Ochsenbein, M. G. Allen, \& D. Egret, 759

Godet, O., Plazolles, B., Kawaguchi, T., et al. 2012, ApJ, 752, 34, doi: 10.1088/0004-637X/752/1/34

Grundstrom, E. D., Blair, J. L., Gies, D. R., et al. 2007, ApJ, 656, 431, doi: 10.1086/510508

Hu, C.-P., Chou, Y., \& Chung, Y.-Y. 2008, ApJ, 680, 1405, doi: $10.1086 / 527549$

Hu, C.-P., Chou, Y., Ng, C.-Y., Lin, L. C.-C., \& Yen, D. C.-C. 2017, ApJ, 844, 16, doi: 10.3847/1538-4357/aa79a3

Hu, C.-P., Chou, Y., Yang, T.-C., \& Su, Y.-H. 2013, ApJ, 773, 58, doi: 10.1088/0004-637X/773/1/58 
Israel, G. L., Belfiore, A., Stella, L., et al. 2017, Science, 355, 817, doi: 10.1126/science.aai8635

Kaaret, P., Feng, H., \& Roberts, T. P. 2017, ARA\&A, 55, 303, doi: 10.1146/annurev-astro-091916-055259

King, A., \& Lasota, J.-P. 2019, MNRAS, 485, 3588, doi: $10.1093 / \mathrm{mnras} / \mathrm{stz} 720$

King, A. R. 2008, MNRAS, 385, L113, doi: 10.1111/j.1745-3933.2008.00444.x

Koliopanos, F., Vasilopoulos, G., Godet, O., et al. 2017, A\&A, 608, A47, doi: 10.1051/0004-6361/201730922

Leahy, D. A., Darbro, W., Elsner, R. F., et al. 1983, ApJ, 266, 160, doi: 10.1086/160766

Li, K. L., Hu, C.-P., Lin, L. C. C., \& Kong, A. K. H. 2016, ApJ, 828, 74, doi: 10.3847/0004-637X/828/2/74

Mushtukov, A. A., Suleimanov, V. F., Tsygankov, S. S., \& Poutanen, J. 2015, MNRAS, 454, 2539, doi: 10.1093/mnras/stv2087

Naik, S., Paul, B., \& Ali, Z. 2011, ApJ, 737, 79, doi: 10.1088/0004-637X/737/2/79

Rodríguez Castillo, G. A., Israel, G. L., Belfiore, A., et al. 2020, ApJ, 895, 60, doi: 10.3847/1538-4357/ab8a44

Sathyaprakash, R., Roberts, T. P., Walton, D. J., et al. 2019, MNRAS, 488, L35, doi: 10.1093/mnrasl/slz086

Scargle, J. D., Norris, J. P., Jackson, B., \& Chiang, J. 2013, ApJ, 764, 167, doi: 10.1088/0004-637X/764/2/167

Stellingwerf, R. F. 1978, ApJ, 224, 953, doi: 10.1086/156444

Stobbart, A. M., Roberts, T. P., \& Warwick, R. S. 2004, MNRAS, 351, 1063, doi: 10.1111/j.1365-2966.2004.07848.x
Straub, O., Godet, O., Webb, N., Servillat, M., \& Barret, D. 2014, A\&A, 569, A116, doi: 10.1051/0004-6361/201423874

Terashima, Y., \& Wilson, A. S. 2004, ApJ, 601, 735, doi: $10.1086 / 380505$

The Astropy Collaboration. 2018a, astropy v3.0.5: a core python package for astronomy, 3.0.5, Zenodo, doi: 10.5281/zenodo. 2556700

—. 2018b, astropy v3.0.5: a core python package for astronomy, 3.0.5, Zenodo, doi: 10.5281/zenodo.1461536

Townsend, L. J., \& Charles, P. A. 2020, MNRAS, 495, 139, doi: $10.1093 / \mathrm{mnrasl} / \mathrm{slaa0} 78$

Trudolyubov, S. P. 2008, MNRAS, 387, L36, doi: 10.1111/j.1745-3933.2008.00478.x

Trudolyubov, S. P., Priedhorsky, W. C., \& Córdova, F. A. 2007, ApJ, 663, 487, doi: 10.1086/518500

Urquhart, R., \& Soria, R. 2016, MNRAS, 456, 1859, doi: $10.1093 / \mathrm{mnras} / \mathrm{stv} 2293$

Vasilopoulos, G., Lander, S. K., Koliopanos, F., \& Bailyn, C. D. 2020, MNRAS, 491, 4949, doi: $10.1093 / \mathrm{mnras} / \mathrm{stz} 3298$

Wang, S., Soria, R., Urquhart, R., \& Liu, J. 2018, MNRAS, 477, 3623, doi: 10.1093/mnras/sty 872

Wilms, J., Allen, A., \& McCray, R. 2000, ApJ, 542, 914, doi: 10.1086/317016

Zhou, Y., Feng, H., Ho, L. C., \& Yao, Y. 2019, ApJ, 871, 115, doi: 10.3847/1538-4357/aaf724 